\title{
Decoupling Control Approach for Neonate Incubator System
}

\author{
Elyes FEKI \\ Department of Physics, Faculty \\ of Science, Mathematics, \\ Physics and Natural of Tunis
}

\author{
M. Aymen ZERMANI \\ Department of Electric \\ Engineering, National School \\ of Engineering of Tunis
}

\author{
Abdelkader MAMI \\ Department of Physics, Faculty \\ of Science, Mathematics, \\ Physics and Natural of Tunis
}

\begin{abstract}
This paper presents an adaptive decoupling temperature and humidity control for neonatal incubator process by exploiting an active humidification system. The neonatal incubator is a Two-Input Two-Output process (TITO) with characteristics of strong coupling and time variation. The coupling problem is treated by the weight adjustment of the output error to reduce the effect of coupling and to enhance control performance. In addition, an Adaptive Decoupling strategy based on Generalized Predictive Control (ADGPC) with Multivariable Recursive Extended Least-Squares (MVRELS) parameters estimator is used. The simulation and real results demonstrate that the decoupling by error dependent tuning of the weighting factor can eliminate the coupling influence with better control performance and can be easily generalized to the Multiple-Input - Multiple-Output (MIMO) systems.
\end{abstract}

\section{General Terms}

Incubator process, Multivariable system, Decoupling Predictive Control.

\section{Keywords}

Incubator process, TITO, GPC, decoupling, adaptive control.

\section{INTRODUCTION}

Many industrial processes have strong cross coupling between control loops. Mostly Proportional Integral Differential (PID) is the simplest solution to control these systems. But these controllers do not offer satisfactory control because of interactions. Also the tuning of PID is complicated when they are used in MIMO systems. Generalized predictive control appears to be attractive solution in multivariable process control $[1,2]$. However, these controllers cannot solve problems with strong interaction. In that case, decoupling can perfectly achieved by adding interaction compensators to controllers [3]. But in some systems because of improper delay structure or model mismatch, interaction compensators cannot applicable [4]. Therefore, a practical method for decoupling is required. The first method use decelerated set point change witch a slower change of the reference signal leads to less coupling effect that recommended by [5]. The second method introduces an additional error signal weighting, where controller observe reference change in a control loop it increases error weights in all other loops that decrease interactions. The incubator process is used to produce healthful micro-environment in order to reduce new born heat loss by controlling temperature inside incubator. Temperature is one of the most important factors that need to be maintained with a minimum variation. But only temperature control is not sufficient to provide comfortable environment. Also, the relative humidity control is very important to reduce the new born heat loss [6,7]. For these reasons, we have developed an active humidification system based on a nebulizer. Therefore, this process becomes a TITO system (Two Input-Two-Output). In fact, the process has time-varying parameters model, depending on the external temperature and on the load under treat. Also, couplings between temperature and humidity are very important that should be reduced. The objective of this paper is to develop an adaptive decoupling GPC for temperature and humidity control of an incubator system with variable power heater and variable-frequency nebulizer control. In fact, the choice of a constant weighting factor on the output error does not allow at the same time to be powerful under control performance and decoupling. This compromise is resolved by the varying weighting factor on the output error. The proposed control method combines the well-known Extended Least Squares parameters method and the DGPC strategy, in order to accomplish satisfactory decoupling and guaranteed stability simultaneously. The paper is structured as follows. The section 2 is devoted to present a multivariable adaptive predictive control. In section 3 , a new tuning procedure of the error weighting factor is developed to eliminate the coupling influence with better control performance. In Section 4 experimental setup and computer simulations are conducted to review the feasibility and effectiveness of the proposed controller, and experimental results are used for illustration of the merit and performance of the controlled incubator system.

\section{THEORETICAL CONSIDERATIONS}

\subsection{Process model}

The synthesis of the generalized predictive controller (GPC) suggested by (Clarke et al, 1987; Clarke, 1988) [1,8]. This method was used successfully in industrial applications of various forms (Richalet et al,1987; Richalet et al,2009) [9,10]. The approach of generalized predictive control is based on a dynamic model of type ARIMAX (Auto Regressive Integrated Moving Average with eXternal inputs), given by:

$$
A_{i}\left(z^{-1}\right) y_{i}(k)=z^{-d_{i}} B_{i}\left(z^{-1}\right) u_{i}(k-1)+C_{i}\left(z^{-1}\right) \frac{e_{i}(k)}{\Delta\left(z^{-1}\right)} .
$$

Where $i$ is the number system, $y_{i}(k)$ is the system output, $u_{i}(k)$ is the system input, $e_{i}(k)$ is the uncorrelated random sequence, $\Delta\left(z^{-1}\right)=1-z^{-1}$ corresponds to an integral action. Its presence in the direct channel allows a zero error in steady state value.

$$
A_{i}\left(z^{-1}\right), B_{i}\left(z^{-1}\right) \text { and } C_{i}\left(z^{-1}\right) \text { are polynomials. }
$$




$$
\begin{aligned}
& A_{i}\left(z^{-1}\right)=1+a_{1} z^{-1}+a_{2} z^{-2}+\cdots+a_{n a i} z^{-n a_{i}}, \\
& B_{i}\left(z^{-1}\right)=b_{0}+b_{1} z^{-1}+b_{2} z^{-2}+\cdots+b_{n b i} z^{-n b_{i}}, \\
& C_{i}\left(z^{-1}\right)=1+c_{1} z^{-1}+c_{2} z^{-2}+\cdots+c_{n c i} z^{-n c i}
\end{aligned}
$$

With $n a_{i}, n b_{i}, n c_{i}$ indicate the respective order of these polynomials.

\subsection{Cost function}

The generalized predictive control based on the minimization of a quadratic criterion on a sliding horizon, which involves a term related to the difference between the predicted output sequence and the sequence of future control [11].

The criterion is given by:

$$
J_{i}=\lambda_{y i} \sum_{t=N_{i}}^{H P_{i}}\left[y_{C_{i}}(k+t)-\hat{y}_{i}(k+t)\right]^{2}+\lambda_{u i} \sum_{t=1}^{N_{c_{i}}} \Delta u_{i}^{2}(k+t-1)(3)
$$

with $\hat{y}_{i}(k)$ is the output value predicted at time $\mathrm{k}, y_{C_{i}}(k)$ is the set points values at time $\mathrm{k}, \Delta u_{i}(k)$ is the increment of control at time $\mathrm{k}, N_{i}$ is the minimum prediction horizon, $H P_{i}$ is the maximum prediction horizon, $N_{C_{i}}$ is the control horizon, $\lambda_{u i}$ is the control weighting factor and $\lambda_{y i}$ is the error weighting factor.

\subsection{Prediction of the system output}

Consider the output expressed by (1) the output at time instant $(k+t)$ will be:

$$
y_{i}(k+t)=\frac{B_{i}\left(z^{-1}\right)}{A_{i}\left(z^{-1}\right)} u_{i}\left(k+t-d_{i}-1\right)+\frac{C_{i}\left(z^{-1}\right)}{A_{i}\left(z^{-1}\right) \Delta\left(z^{-1}\right)} e_{i}(k+t)
$$

By applying the Euclidean algorithm on the second term of (4) we get

$$
\frac{C_{i}\left(z^{-1}\right)}{A_{i}\left(z^{-1}\right) \Delta\left(z^{-1}\right)}=L_{t}\left(z^{-1}\right)+z^{-t} \frac{G_{t}\left(z^{-1}\right)}{A_{i}\left(z^{-1}\right) \Delta\left(z^{-1}\right)}
$$

After using (4) and (5), we assume that the term related to the disturbance is zero, the optimal predictor of the output is:

$$
\hat{y}_{i}(k+t)=\frac{L_{t}\left(z^{-1}\right) B_{i}\left(z^{-1}\right) \Delta\left(z^{-1}\right)}{C_{i}\left(z^{-1}\right)} u_{i}\left(k+t-d_{i}-1\right)+\frac{G_{t}\left(z^{-1}\right)}{C_{i}\left(z^{-1}\right)} y_{i}(k)
$$

A second Diophantine equation decompose the predictor in two terms: a term based on the current output, old orders, the system output and a second term dependent on future orders.

$$
\frac{\sigma_{i}\left(z^{-1}\right)}{C_{i}\left(z^{-1}\right)}=H_{t}\left(z^{-1}\right)+z^{-t+d_{i}} \frac{R_{t}\left(z^{-1}\right)}{C_{i}\left(z^{-1}\right)}
$$

with

$$
\sigma_{i}\left(z^{-1}\right)=L_{t}\left(z^{-1}\right) B_{i}\left(z^{-1}\right)
$$

The optimal predictor of the output is:

$$
\begin{aligned}
\hat{y}_{i}(k+t) & =H_{t}\left(z^{-1}\right) \Delta\left(z^{-1}\right) u_{i}\left(k+t-d_{i}-1\right)+ \\
& \frac{G_{t}\left(z^{-1}\right)}{C_{i}\left(z^{-1}\right)} y_{i}(k)+\frac{R_{t}\left(z^{-1}\right)}{C_{i}\left(z^{-1}\right)} \Delta\left(z^{-1}\right) u_{i}(k-1)
\end{aligned}
$$

Where $H_{t}\left(z^{-1}\right) G_{t}\left(z^{-1}\right) R_{t}\left(z^{-1}\right)$ et $L_{t}\left(z^{-1}\right)$ are polynomial solutions to the Diophantine equations [1]. The matrix formulation is represented by:

$$
\hat{Y}_{i}=\hat{H}_{i} \Delta U_{i}+\hat{G}_{i} Y_{i}^{*}+\hat{R}_{i} \Delta U_{i}^{*}
$$

The vector of the predicted outputs $\hat{Y}_{i}$ is the sum of the predicted forced $\hat{H}_{i} \Delta U_{i}$ and free responses $\hat{G}_{i} Y_{i}^{*}+\hat{R}_{i} \Delta U_{i}^{*}$ with:

$$
\begin{aligned}
& \hat{Y}_{i}=\left[\hat{y}_{i}(k+1 / k), \hat{y}_{i}(k+2 / k) \cdots \hat{y}_{i}\left(k+H P_{i} / k\right)\right]^{T}, \\
& Y_{i}^{*}=\left[y_{i}^{*}(k), y_{i}^{*}(k-1) \cdots y_{i}^{*}\left(k-n a_{i}\right)\right]^{T}, \\
& \Delta U_{i}=\left[\Delta u_{i}(k) \cdots \Delta u_{i}\left(k+N_{C i}-1\right)\right]^{T}, \\
& \Delta U_{i}^{*}=\left[\Delta u_{i}^{*}(k-1) \cdots \Delta u_{i}^{*}(k-n b i-d i+1)\right]^{T}, \\
& \hat{G}_{i}=\left[G_{1+d_{i}}\left(z^{-1}\right) \cdots G_{H P_{i}+d_{i}}\left(z^{-1}\right)\right]^{T}, \\
& \hat{R}_{i}=\left[R_{1+d_{i}}\left(z^{-1}\right) \cdots R_{H P_{i}+d_{i}}\left(z^{-1}\right)\right]^{T}, \\
& \hat{H}_{i}=\left(\begin{array}{cccc}
h_{0} & 0 & \cdots & 0 \\
h_{1} & h_{0} & \cdots & 0 \\
\vdots & \vdots & \vdots & \vdots \\
h_{H P_{i}-d i-1} & h_{H P_{i}-d i-2} & \cdots & h_{H P_{i}-d i-N_{C i}}
\end{array}\right)
\end{aligned}
$$

where

$y_{i}^{*}(k)=\frac{y_{i}(k)}{C_{i}\left(z^{-1}\right)}, \Delta u_{i}^{*}(k-1)=\frac{\Delta u_{i}(k-1)}{C_{i}\left(z^{-1}\right)}$,

$$
\text { and }\left\{\begin{array}{l}
\operatorname{DIM}\left(\hat{G}_{i}\right)=\left(\mathrm{HP}_{i}-\mathrm{d}_{i}, \mathrm{na}_{i}+\mathrm{d}_{i}-1\right) \\
\operatorname{DIM}\left(\hat{R}_{i}\right)=\left(\mathrm{HP}_{i}-\mathrm{d}_{i}, \mathrm{nb}_{i}+\mathrm{d}_{i}-1\right) \\
\operatorname{DIM}\left(\hat{H}_{i}\right)=\left(\mathrm{HP}_{i}-\mathrm{d}_{i}, \mathrm{~N}_{C i}\right)
\end{array}\right.
$$

denote the dimension of $\hat{G}_{i}, \hat{R}_{i}$ and $\hat{H}_{i}$, respectively.

\subsection{Law order}

We write the criterion $\mathrm{J}$ in matrix form

$$
\begin{aligned}
& \qquad J_{i}=\left[\hat{Y}_{i}(k)-Y_{C_{i}}(k)\right]^{T} \chi_{i}\left[\hat{Y}(k)-Y_{C_{i}}(k)\right]+\lambda_{u i} \Delta U_{i}(k)^{T} \Delta U_{i}(k) \\
& \text { with } Y_{C i}=\left[y_{C_{i}}\left(k+d_{i}\right) \cdots y_{C i}\left(k+H P_{i}+d_{i}\right)\right]
\end{aligned}
$$

The optimal vector $\Delta U_{i}$ is

$$
\Delta U_{i}=\left(\hat{H}_{i}^{T} \chi_{i} \hat{H}_{i}+\lambda_{u i} I_{N_{C i}}\right)^{-1} \hat{H}_{i}^{T} \hat{Y}_{i}^{T}
$$


The optimal control law is derived from analytical minimization of the previous cost function. Only the first control value is finally applied to the system.

$$
u_{i}(k)=\Delta u_{i}(k)+u_{i}(k-1)
$$

Which $\Delta u_{i}(k)$ is the first element of the vector $\Delta U_{i}$ and $I_{N_{C}}$ is diagonal matrix of size $N_{C i} * N_{C i}$ and $\chi_{i}$ is diagonal matrix of size $H P_{i}-d_{i} * H P_{i}-d_{i}$.

$$
I_{N_{C i}}=\left(\begin{array}{ccc}
1 & & 0 \\
& \ddots & \\
0 & & 1
\end{array}\right), \chi_{i}=\left(\begin{array}{ccc}
\lambda_{y i} & & 0 \\
& \ddots & \\
0 & & \lambda_{y i}
\end{array}\right)
$$

For the system with constrains on the controller output value, on the controller increment output value or on the system output value, the vector $\Delta u_{i}$ is calculated by function FMINCON of Optimization Toolbox of the language Matlab. We can express constraint on the process in the form

$$
\begin{aligned}
& u_{\min } \leq u_{i}(k) \leq u_{\max } \\
& \Delta u_{\min } \leq \Delta u_{i}(k) \leq \Delta u_{\max }
\end{aligned}
$$

\subsection{Adaptive control}

In this part, our aim is to describe the design strategy of the Adaptive Predictive Controller (AGPC) [12-14] for incubator system. The synthesis of the previous predictive control considers that the parameters of the process are fixed, but in reality this is not the case. In fact, the incubator has timevarying parameters model, depending on the external temperature and on the load under treat. The Multi Variable Recursive Extended Least Squares algorithm (MVRELS) is applied to estimate the unknown system parameters $\hat{A}_{i j}$ and $\hat{B}_{i j}$ and the estimated parameters are updated at each sampling period for tuning of the GPC control $\hat{H}_{t}, \hat{G}_{t}, \hat{R}_{t}$ and $\hat{L}_{j}$.

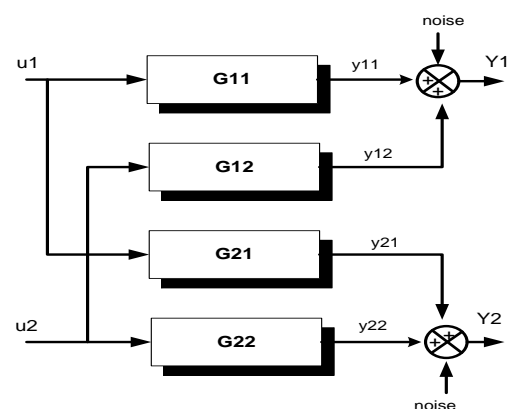

Fig1:TITO process model.

The transfer functions of the various subsystems are described by

$$
y_{i j}(k)=\frac{z^{-d i j} B_{i j}\left(z^{-1}\right)}{A_{i j}\left(z^{-1}\right)} u_{i}(k-1)
$$

Where $i$ and $j$ are the level indices $(i, j=1,2)$ and the two equations that represent the two outputs of the systems are

$$
\begin{aligned}
& y_{1}(k)=y_{11}(k)+y_{12}(k)+\xi_{1}, \\
& y_{2}(k)=y_{22}(k)+y_{21}(k)+\xi_{2} . \\
& y_{1}(k)=\frac{z^{-d 11} B_{11}\left(z^{-1}\right) A_{12}\left(z^{-1}\right)}{A_{11}\left(z^{-1}\right) A_{12}\left(z^{-1}\right)} u_{1}(k-1)+ \\
& \frac{z^{-d 12} B_{12}\left(z^{-1}\right) A_{11}\left(z^{-1}\right)}{A_{12}\left(z^{-1}\right) A_{11}\left(z^{-1}\right)} u_{2}(k-1)+C_{1}\left(z^{-1}\right) \frac{e_{1}(k)}{A_{12}\left(z^{-1}\right) A_{11}\left(z^{-1}\right)}, \\
& y_{2}(k)=\frac{z^{-d 22} B_{22}\left(z^{-1}\right) A_{21}\left(z^{-1}\right)}{A_{22}\left(z^{-1}\right) A_{21}\left(z^{-1}\right)} u_{2}(k-1) \\
& +\frac{z^{-d 21} B_{21}\left(z^{-1}\right) A_{22}\left(z^{-1}\right)}{A_{21}\left(z^{-1}\right) A_{22}\left(z^{-1}\right)} u_{1}(k-1)+C_{2}\left(z^{-1}\right) \frac{e_{2}(k)}{A_{21}\left(z^{-1}\right) A_{22}\left(z^{-1}\right)}
\end{aligned}
$$

Also, we can rewrite $y_{1}$ and $y_{2}$ as follow:

$$
\begin{aligned}
y_{1}(k)= & \frac{z^{-d 11} P_{11}\left(z^{-1}\right)}{D_{11}\left(z^{-1}\right)} u_{1}(k-1)+\frac{z^{-d 12} P_{12}\left(z^{-1}\right)}{D_{11}\left(z^{-1}\right)} u_{2}(k-1)+ \\
& C_{1}\left(z^{-1}\right) \frac{e_{1}(k)}{D_{11}\left(z^{-1}\right)}, \\
y_{2}(k)= & \frac{z^{-d 22} P_{22}\left(z^{-1}\right)}{D_{22}\left(z^{-1}\right)} u_{2}(k-1)+\frac{z^{-d 21} P_{21}\left(z^{-1}\right)}{D_{22}\left(z^{-1}\right)} u_{1}(k-1)+ \\
& C_{2}\left(z^{-1}\right) \frac{e_{2}(k)}{D_{22}\left(q^{-1}\right)}
\end{aligned}
$$

Based on the expression (27) of the global outputs $y_{i}$, we can estimate coefficients of the polynomials $D_{11}, D_{22}, P_{11}, P_{12}, P_{21}, P_{22}$ using the MVRELS technique,

with $n d 1, n d 2, n p 11, n p 12, n p 21, n p 22$ indicate the respective order of these polynomials

The MVRELS estimation method with forgetting factor $\lambda_{1}$ and $\lambda_{2}$ is expressed by:

$$
\begin{aligned}
& P(k)=\frac{1}{\lambda_{1}}\left[P(k-1)-\frac{P(k-1) \varphi_{i}^{T}(k) P(k-1)}{\frac{\lambda_{1}}{\lambda_{2}}+\varphi_{1}(k) \varphi_{i}^{T}(k) P(k-1)}\right], \\
& K(k)=P(k) \varphi_{i}(k), \\
& \theta_{i}(k)=\theta_{i}(k-1)+K(k)\left(y_{i}(k)-\varphi_{i}(k)^{T} \theta_{i}(k-1)\right)
\end{aligned}
$$

where $\mathrm{i}=1$

$$
\begin{aligned}
\varphi_{1}(k)= & {\left[y_{1}(k-1) \cdots y_{1}(k-n d 1), u_{1}\left(k-d_{11}-1\right) \cdots u_{1}\left(k-d_{11}-n p_{11}\right),\right.} \\
& \left.u_{2}\left(k-d_{12}-1\right) \cdots u_{2}\left(k-d_{12}-n p_{12}\right), e_{1}(k-1) \cdots e_{1}\left(k-n c_{1}\right)\right]^{T} \\
\theta_{1}(k)=[ & {\left[D^{11}{ }_{1} \cdots D^{11}{ }_{n d 1}, P^{11}{ }_{1} \cdots P^{11}{ }_{n p 11}, P^{12}{ }_{1} \cdots P^{12}{ }_{n p 12}, c_{1} \cdots c_{n c 1}\right]^{T} }
\end{aligned}
$$


where $\mathrm{i}=2$

$\varphi_{2}(k)=\left[y_{2}(k-1) \cdots y_{2}(k-n d 1), u_{2}\left(k-d_{22}-1\right) \cdots u_{2}\left(k-d_{22}-n p_{22}\right)\right.$,

$\left.u_{1}\left(k-d_{21}-1\right) \cdots u_{1}\left(k-d_{21}-n p_{21}\right), e_{2}(k-1) \cdots e_{2}\left(k-n c_{1}\right)\right]^{T}$

$\theta_{2}(k)=\left[D_{1}^{22} \cdots D_{n d 2}^{21}, P_{1}^{22} \cdots P_{n p 22}^{22}, P_{1}^{21} \cdots P_{n p 21}^{21}, c_{1} \cdots c_{n c 2}\right]^{T}$

$K(k)$ is an error correct gain vector; $P(k)$ is symmetrical matrix; $\lambda$ is a forgetting factor. Noise $e_{i}$ cannot be measured. However, we can make a good estimation of $\hat{\boldsymbol{e}}_{i}(k)$ by $\hat{e}_{i}(k)=y_{i}(k)-\varphi_{i}(k)^{T} \theta_{i}(k)$

\section{DECOUPLING DESIGN OF GPC}

\subsection{Decoupling by decelerating the}

\section{reference signal change}

In order to solve the problem of coupling, a TITO process model Fig 1 is considered. The two output variables $\left(y_{1}, y_{2}\right)$ of the process become the controlled variables; the two variable inputs $\left(\boldsymbol{u}_{1}, \boldsymbol{u}_{2}\right)$ are the manipulated variables $\xi_{1}$ and $\xi_{2}$ are non-measurable disturbances. Assume the system to be controlled is a double-input/double-outputs system.

$$
\begin{aligned}
& A\left(z^{-1}\right) Y(k)=B\left(z^{-1}\right) U(k-1)+\xi(k), \\
& A\left(z^{-1}\right)=\left[\begin{array}{cc}
1-1.4 z^{-1}+0.45 z^{-1} & 0 \\
0 & 1-1.2 z^{-1}+0.32 z^{-1}
\end{array}\right], \\
& B\left(z^{-1}\right)=\left[\begin{array}{cc}
0.209 z^{-1}+z^{-2} & 0.213-0.25 z^{-1} \\
0.226 z^{-1}+0.324 z^{-2} & 0.131+0.109 z^{-1}
\end{array}\right], \\
& \xi=\left[\begin{array}{l}
\xi_{1} \\
\xi_{2}
\end{array}\right], U=\left[\begin{array}{l}
u_{1} \\
u_{2}
\end{array}\right], Y=\left[\begin{array}{l}
y_{1} \\
y_{2}
\end{array}\right]
\end{aligned}
$$

We write the criterion $\mathrm{J}$ of each controller

$$
\begin{aligned}
& \text { GPC1: } J_{1}=\lambda_{y 1} \sum_{j=N_{1}}^{H P_{1}}\left[y_{C_{1}}(k+t)-\hat{y}_{1}(k+t)\right]^{2}+ \\
& \lambda_{u 1} \sum_{j=1}^{N_{C}} \Delta u_{1}^{2}(k+t-1), \\
& \text { GPC2: } J_{2}=\lambda_{y 2} \sum_{j=N_{2}}^{H P_{2}}\left[y_{C_{2}}(k+t)-\hat{y}_{2}(k+t)\right]^{2}+ \\
& \lambda_{u 2} \sum_{j=2}^{N_{C}} \Delta u_{2}^{2}(k+t-1) \text {, }
\end{aligned}
$$

It is clear that a slower change of the reference signal leads to less coupling effect $[5,15]$. This is proved in Fig 2 and in Fig3.

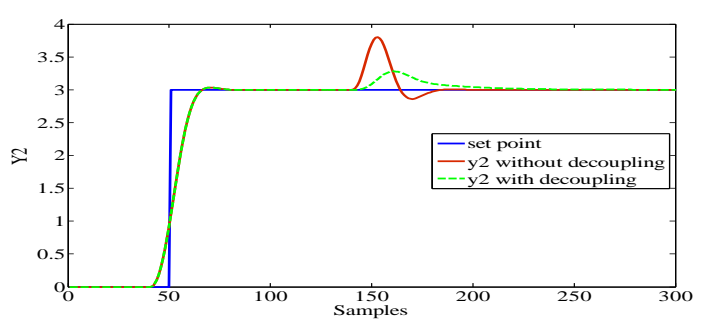

Fig 2: Evolution of the output y2 with and without decoupling by decelerating the reference signal.

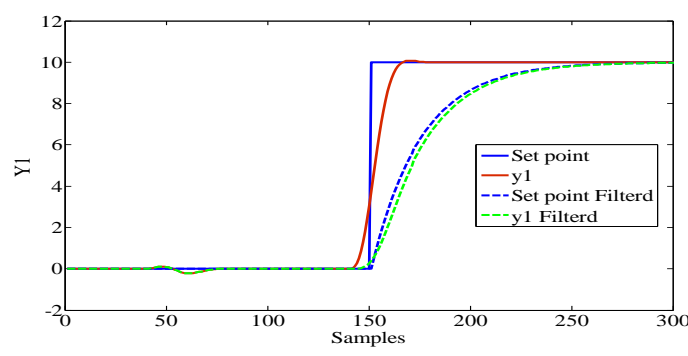

Fig 3: Evolution of the output y1 to input unit steps with and without filtered.

The stepwise change of the set-value was filtered by a firstorder filter. The filter parameters were selected in such a way that the effect of coupling will be reduced.

$$
Y_{C_{-} \text {filtred }}=\frac{b z^{-1}}{1-a z^{-1}} Y_{C_{i}}
$$

The use of a filter on the reference signal reduces the coupling effect but at the cost of the desired performance.

\subsection{Decoupling by tuning error weighting factor}

In order to present the decoupling approach proposed, the TITO system (31) is used, and the structure of the decoupling control is also described in Fig 4.The main idea of decoupling controller is the following: The error weight factor of the output variable whose reference is kept constant should be increased and synchronized with the variable whose reference is modified. We note that a high value of the weighting factor $\lambda_{\max }$ on output error can generate a very drastic change in controlled variables and degrades the control performance, what we are trying to avoid in most cases. On the other hand, a low value of weighting factor $\lambda_{\min }$ does not allow reducing the effect of the coupling caused by the other variable.

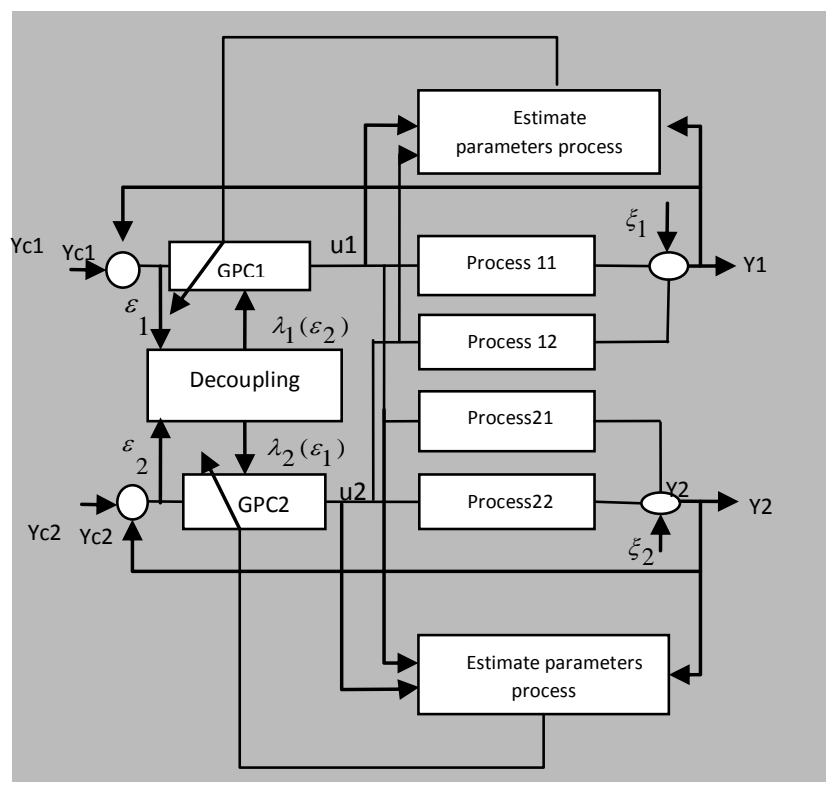

Fig 4: Control structure of adaptive generalized predictive control with weighting factor adjusting 
To satisfy some performance (smooth control movements and minimum coupling effect), we establish the weighting factors expressions

$$
\begin{aligned}
& \lambda_{y 1}=\left\{\begin{array}{cc}
\lambda_{\max 1} & \lambda_{y 1} \geq \lambda_{\max 1} \\
\lambda_{\min 1}+\exp \left(\left|\varepsilon_{2}(k)\right|\right) & \lambda_{y 1} \leq \lambda_{\max 1}
\end{array}\right\}, \\
& \lambda_{y 2}=\left\{\begin{array}{cc}
\lambda_{\max 2} & \lambda_{y 2} \geq \lambda_{\max 2} \\
\lambda_{\min 2}+\exp \left(\left|\varepsilon_{1}(k)\right|\right) & \lambda_{y 2} \leq \lambda_{\max 2}
\end{array}\right\}
\end{aligned}
$$

with $\varepsilon_{1}(k)=y_{C_{1}}(k)-y_{1}(k), \quad \varepsilon_{2}(k)=y_{C_{2}}(k)-y_{2}(k)$.

A good decoupling with better control performance seemed to be optimal when an exponential change of the weighting factors is used and the limit value is respected. Tuning of $\lambda_{y_{i}}$ depends on process parameters [15] and can vary in broad range $[1,1000]$. Higher $\lambda_{\text {max }}$ leads to better decoupling. To check the validation of decoupling designed a simulation results are presented in Fig. 5 and Fig. 6. It should be mentioned that control horizon is $N_{C_{1}}=N_{C_{2}}=1$ and the control prediction is $H P_{1}=H P_{2}=15$. Weighting of the control error for the first and second manipulated variables are: $\lambda_{y 1}=\left\{\begin{array}{cc}50 & \lambda_{y 1} \geq 50 \\ 1+\exp \left(\left|\varepsilon_{2}(k)\right|\right) & \lambda_{y 1} \leq 50\end{array}\right\}, \lambda_{y 2}=\left\{\begin{array}{cc}50 & \lambda_{y 2} \geq 50 \\ 1+\exp \left(\left|\varepsilon_{1}(k)\right|\right) & \lambda_{y 2} \leq 50\end{array}\right\}$

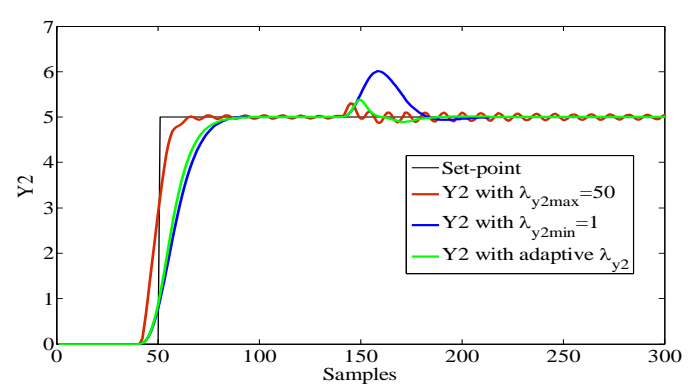

Fig 5: Evolution of the outputs y2 with and without decoupling .

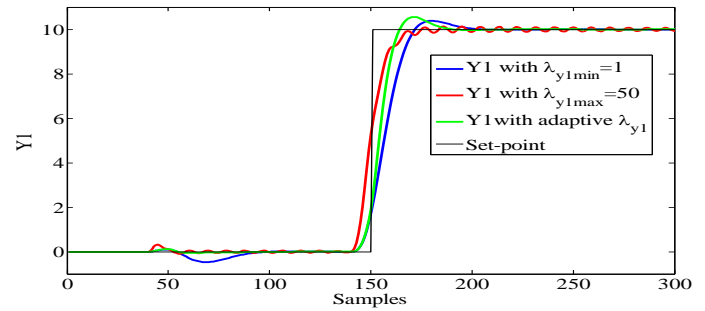

Fig 6: Evolution of the outputs y1 with and without decoupling.

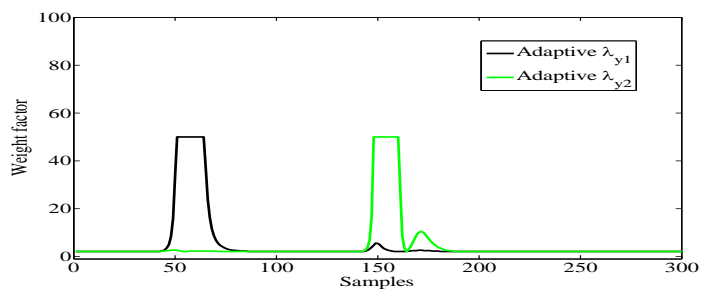

Fig 7: Evolution of the weighting factor $\lambda_{y 1}$ and $\lambda_{y 2}$.
Fig 5 and Fig 6 show that the choice of a constant weighting factor on the output error does not allow at the same time to be powerful under better control performance and decoupling. This compromise is resolved by the varying weighting factor on the output error $\lambda_{y i}$ with using (35).

\section{SIMULATION, EXPERIMENTAL RESULTS AND DISCUSSIONSS}

This section emphasizes on examining the performance of the proposed Adaptive Decoupling Generalized Predictive Controller (ADGPC) for temperature and humidity control of a new born incubator system.

\subsection{Experiment setup}

A neonatal incubator is, usually, a small (approx- imately: 0.5 $\mathrm{x} 0.5 \times 1 \mathrm{~m}^{3}$ ) cabinet with transparent walls so that the infant can be easily observed.

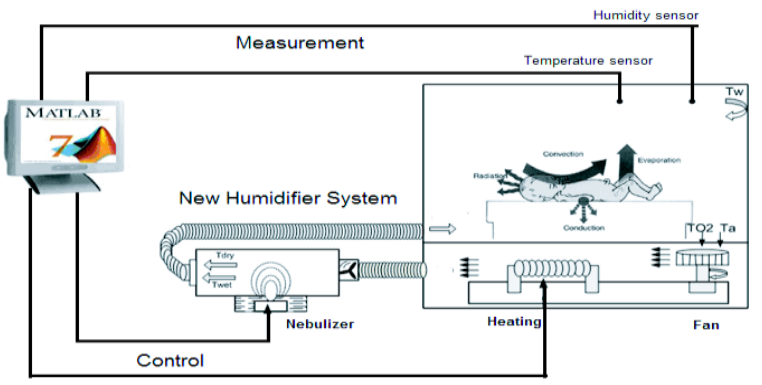

Fig 8: Schematic of the incubator process with experimental arrangement for active humidification to control temperature and Humidity.

The device may include an AC-powered heater, a fan to circulate the warmed air, a container for water to add humidity and access ports for nursing care. With the technology available currently, incubators use microprocessor-based control systems to create and to maintain the ideal microclimate for the preterm neonate [16]. The newest incubators, such as the Drager Isolette C2000, use a PID control algorithm to drive the servo control system (Drager Medical Systems, 2004)[17]. Furthermore, the current commercial devices use a passive humidification system, which humidity produced evaporation of water by heating it in the water container [18]. But the passive humidifier cannot provide a high humidity level at low temperature such as in the range of $\left[20^{\circ} \mathrm{C}\right.$ to $\left.38^{\circ} \mathrm{C}\right]$. In this work, we recovered an incubator from Maternal and Neonatal Unit of Rabta-Tunisia, Fig.9. Then, we replaced the passive humidifier by an external block based on a ultrasonic nebulizer system which is an instrument for converting a liquid into a fine spray. This system is able to increase the humidity to $80 \%$. Also we developed a microcontroller-based system devoted to control the humidity and the heating of the new born incubator. The system developed measures the air temperature and the humidity by two sensors LM35 and SY-230. Then, these data are exported to a microcomputer to be analyzed [19]. The aim of this study is to design and implement a closed loop control system to regulate the temperature and humidity inside a neonatal incubator. The presence of coupling makes it impossible to set one without affecting the other. To eliminate the couplings between temperature and humidity, we propose a multivariable decoupled control by tuning the weighting factor. 


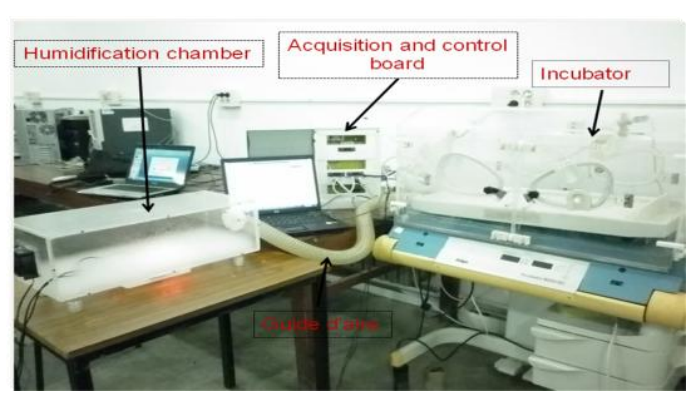

Fig 9: Schematic illustration of the experimental unit's.

\subsection{Identification of process model}

Before proceeding with the real-time control, it is necessary to pass through the modeling system. For this reason, an experimental method is proposed for modeling of the TITO system. The incubator system has Two-Inputs and TwoOutputs.

The inputs to the system are :

$u_{1}$ : control signal applied to the heater,

$u_{2}$ : control signal applied to the nebulizer.

The outputs are:

$y_{1}$ : temperature value output signal,

$y_{2}$ : humidity level output signal.

We define each sub system in Fig 9 by Auto Regressive Integrated Moving Average with eXternal inputs, (ARIMAX) model [20].

$$
y_{i j}(k)=z^{-d i j} \frac{B_{i j}\left(z^{-1}\right)}{A_{i j}\left(z^{-1}\right)} u_{i}(k-1)+\frac{C_{i}\left(z^{-1}\right)}{A_{i j}\left(z^{-1}\right) \Delta\left(z^{-1}\right)} e_{i}(k)
$$

Where $d_{i j}$ is time delay, $e_{i}$ is white noise and $A_{i j}, B_{i j}, C_{i}$ are:

$$
\begin{aligned}
& A_{i j}\left(z^{-1}\right)=1+a_{1} z^{-1}+a_{2} z^{-2}+\cdots+a_{n a} z^{-n a}, \\
& B_{i j}\left(z^{-1}\right)=b_{0}+b_{1} z^{-1}+b_{2} z^{-2}+\cdots+b_{n b} z^{-n b}, \\
& C_{i}\left(z^{-1}\right)=1+c_{1} z^{-1}+c_{2} z^{-2}+\cdots+c_{n c} z^{-n c} .
\end{aligned}
$$

We present the multivariable system as matrix form:

$$
\left[\begin{array}{l}
y_{1}(k) \\
y_{2}(k)
\end{array}\right]=\left[\begin{array}{ll}
G_{11}(z) & G_{12}(z) \\
G_{21}(z) & G_{22}(z)
\end{array}\right]\left[\begin{array}{l}
u_{1}(k) \\
u_{2}(k)
\end{array}\right]+\left[\begin{array}{l}
\xi_{1} \\
\xi_{2}
\end{array}\right]
$$

The transfer functions (38) are obtained by means of experimental tests through the input and the output data collection. For multivariable open loop experiments, the usual practice is to apply mutually independent Pseudo-Random Binary Signals (PRBSs) to all the manipulated variables of the plant. All experimental data are recorded with sampling times of 20 seconds. A set of models is developed by setting the maximum number of poles $n a$ to $4, n b$ of zeros to 4 , the maximum order of the polynomial perturbation nc to 4 and the maximum time delay nk to 50. The development of all models is achieved by combining the coefficients $n a, n b, n c$ and $n k$. The selection of the appropriate orders of the ARIMAX model is determined according to a validation criterion proposed by [21] which is based on the analysis of the prediction error and the variance of the measured output.

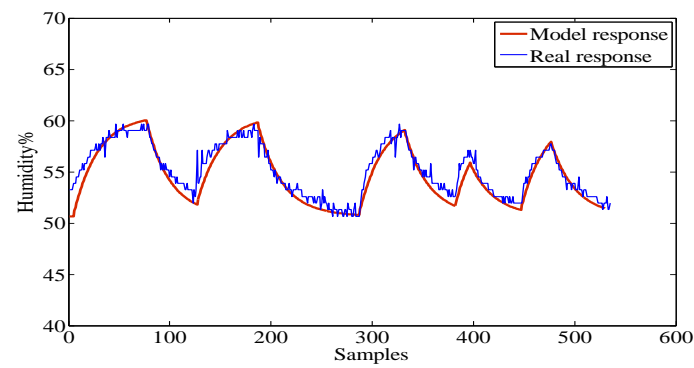

Fig 10: Real and estimated humidity G22.

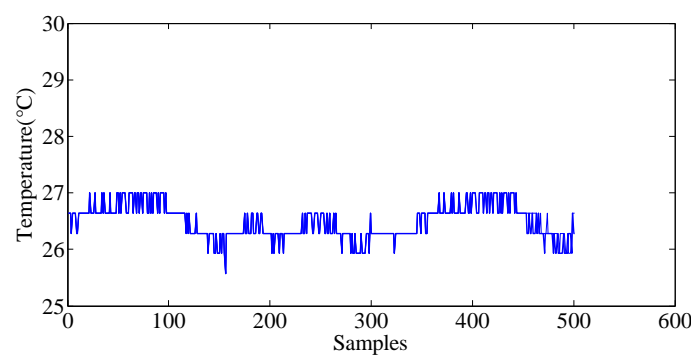

Fig 11: Real temperature G12.

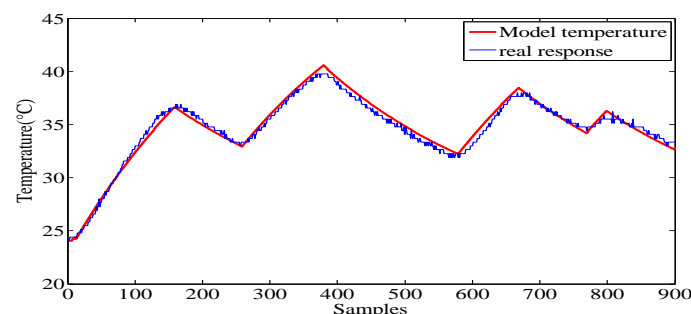

Fig 12: Real and estimated temperature G11.

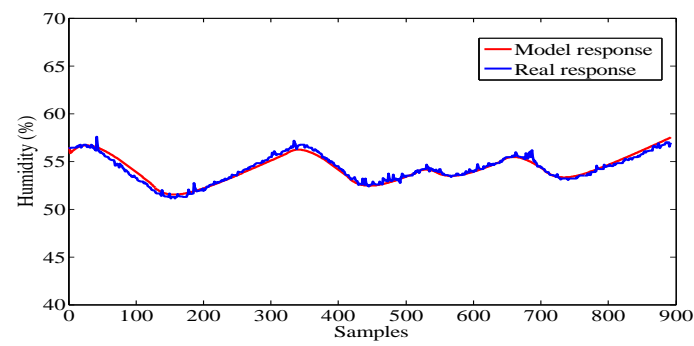

Fig 13: Real and estimated humidity G21.

The experimental results allow to write the transfer function matrix as follows:

$$
\begin{aligned}
& G(z)=\left[\begin{array}{ll}
G_{11} & G_{12} \\
G_{21} & G_{22}
\end{array}\right]=\left[\begin{array}{cc}
z^{-d 11} \frac{B_{11}\left(z^{-1}\right)}{A_{11}\left(z^{-1}\right)} & z^{-d 12} \frac{B_{12}\left(z^{-1}\right)}{A_{11}\left(z^{-1}\right)} \\
z^{-d 21} \frac{B_{21}\left(z^{-1}\right)}{A_{21}\left(z^{-1}\right)} & z^{-d 22} \frac{B_{22}\left(z^{-1}\right)}{A_{22}\left(z^{-1}\right)}
\end{array}\right] \\
& =\left[\begin{array}{cc}
\frac{z^{-10}\left(5.9746 \mathrm{e}-004+5.9412 \mathrm{e}-004 z^{-1}\right)}{1-0.3467 z^{-1}-0.6463 z^{-2}} & 0 \\
\frac{z^{-32}\left(1.3698 \mathrm{e}-004-4.1930 \mathrm{e}-004 z^{-1}\right)}{1-0.28321 z^{-1}-0.7133 z^{-2}} & \frac{z^{-3}\left(0.00203+0.00088 z^{-1}\right)}{1-0.5091 z^{-1}-0.4262 z^{-2}}
\end{array}\right]
\end{aligned}
$$


From Fig 11, the temperature variation is almost not related to the change in moisture level $\left(\mathrm{G}_{12}=0\right)$, and this weak relation can be modeled as a disturbance to the system. The other components are described by a second order system with a time delay. Also, we can rewrite $\mathrm{G}(\mathrm{z})$ in such a way we will have the same denominator for $G_{22}$ and $G_{21}$

$$
\mathrm{G}(\mathrm{z})=\left[\begin{array}{cc}
\mathrm{z}^{-\mathrm{d} 11} \frac{\mathrm{B}_{11}\left(\mathrm{z}^{-1}\right)}{\mathrm{A}_{11}\left(\mathrm{z}^{-1}\right)} & 0 \\
\frac{\mathrm{z}^{-\mathrm{d} 21} \mathrm{P}_{21}\left(\mathrm{z}^{-1}\right)}{\mathrm{D}_{22}\left(\mathrm{z}^{-1}\right)} & \frac{\mathrm{z}^{-\mathrm{d} 22} \mathrm{P}_{22}\left(\mathrm{z}^{-1}\right)}{\mathrm{D}_{22}\left(\mathrm{z}^{-1}\right)}
\end{array}\right]
$$

where $z^{-d 11} \frac{B_{11}\left(z^{-1}\right)}{A_{11}\left(z^{-1}\right)}=\frac{z^{-10}\left(5.9746 e-004+5.9412 \mathrm{e}-004 z^{-1}\right)}{1-0.3467 z^{-1}-0.6463 z^{-2}}$,

$\frac{\mathrm{z}^{-122} \mathrm{P}_{22}\left(\mathrm{z}^{-1}\right)}{\mathrm{D}_{22}\left(\mathrm{z}^{-1}\right)}=\frac{\mathrm{z}^{-3}\left(0.00203+0.00088 \mathrm{z}^{-1}\right)\left(1-0.28321 \mathrm{z}^{-1}-0.7133 \mathrm{z}^{-2}\right)}{\left(1-0.5091 \mathrm{z}^{-1}-0.4262 \mathrm{z}^{-2}\right)\left(1-0.28321 \mathrm{z}^{-1}-0.7133 \mathrm{z}^{-2}\right)}$,

$\frac{\mathrm{z}^{-\mathrm{d} 21} \mathrm{P}_{21}\left(\mathrm{z}^{-1}\right)}{\mathrm{D}_{22}\left(\mathrm{z}^{-1}\right)}=\frac{\mathrm{z}^{-32}\left(1.3698 \mathrm{e}-004-4.1930 \mathrm{e}-004 \mathrm{z}^{-1}\right)\left(1-0.5091 \mathrm{z}^{-1}-0.4262 \mathrm{z}^{-2}\right)}{\left(1-0.28321 \mathrm{z}^{-1}-0.7133 \mathrm{z}^{-2}\right)\left(1-0.5091 \mathrm{z}^{-1}-0.4262 \mathrm{z}^{-2}\right)}$

This structure of the model is used as a start up model for an adaptive GPC synthesis using MVRELS which is described in section 2.5 .

\subsection{Computer simulations and discussions}

Before proceeding with the real time control, it is important to conduct computer simulation with Matlab software to check the feasibility of the proposed approach control. The principal parameters are set as follows: the forgotten factor $\lambda_{1}, \lambda_{2}$ of the MVRELS method are respectively 0.999,1. The prediction horizons are $H P_{1}=40, H P_{2}=20$, the control horizons are $N c_{1}=N c_{2}=1$ and the weighting factors of the control increments are $\lambda_{u 1}=4.1686, \lambda_{u 2}=0.4712$. The coupling effect was suppressed by the method of changing the weighting factor as a function of the control error. A good decoupling could be achieved by the same dependence of the weighting factors as in equation (35) with

$$
\lambda_{y 1}=100 \text { and } \lambda_{y 2}=\left\{\begin{array}{cc}
50 & \lambda_{y 2} \geq 50 \\
1+\exp \left(\left|\varepsilon_{1}(k)\right|\right) & \lambda_{y 2} \leq 50
\end{array}\right\}
$$

In Fig 16 we obtained the ranges of the control signal applied to the heater and to the nebulizer that scaled between $0 \%$ and $100 \%$. The multivariable control is simulated with constraints and at sampling times $\mathrm{T}=20$ seconds. Chosen the inputs constraints: $0 \% \leq u_{1} \leq 100 \%, 0 \% \leq u_{2} \leq 100 \%$.

The described model (39) has strong interaction between input $\mathrm{u} 1$ and output $\mathrm{y} 2$. For this reason, the analyze has been done by changing temperature's reference and observing output signal of the humidity with low, high and adaptive error weighting factor $\lambda_{y 2}$. For $\lambda_{y 1}$, it remains constant and justified by the low interaction between $u_{2}$ and $G_{11}$. In Fig 14 , we present the simulation result of set-point tracking temperature which increases from initial temperature to $26^{\circ} \mathrm{C}$ then to $30^{\circ} \mathrm{C}$ and decreases to $27^{\circ} \mathrm{C}$. In Fig 15, it can be noticed that, there are great differences in the R.H. (Relative Humidity) response due to the choice of the error weighting factor. It is clear that a small constant value of this factor does not reduce the effect of coupling, for against a large value of this latter provides better decoupling at the cost of the quality of the control signal. This compromise is achieved by a choice of error weighting factor with adjustable set point change of the temperature which assures a good decoupling and acceptable control signal. In Fig 16, we present the evolution of the weighting factor with small $\lambda_{y 2 \min }=1$, high $\lambda_{y 2 \max }=20$ and adaptive $\lambda_{y 2}$. In Fig 17, we note that the control signal which corresponds to the choice of adaptive weighting factor presents less excitation than a high value $\lambda_{y 2 \max }=20$.

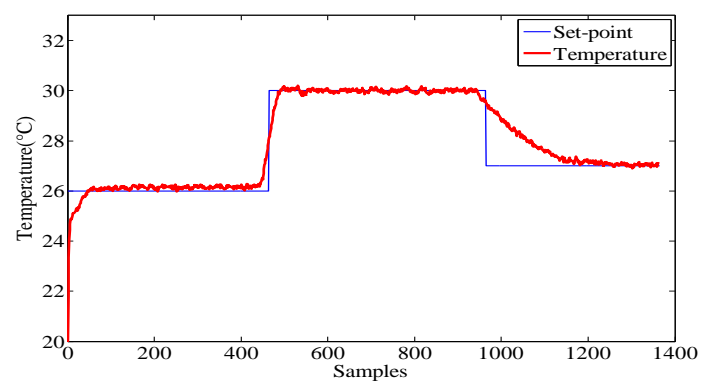

Fig 14: Simulation result of set-point tracking temperature for predictive decoupling control.

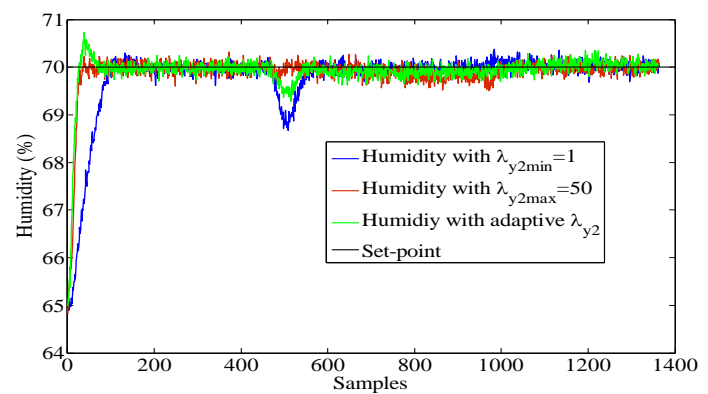

Fig 15:Simulation result of set-point tracking humidity for predictive control with and without decoupling.

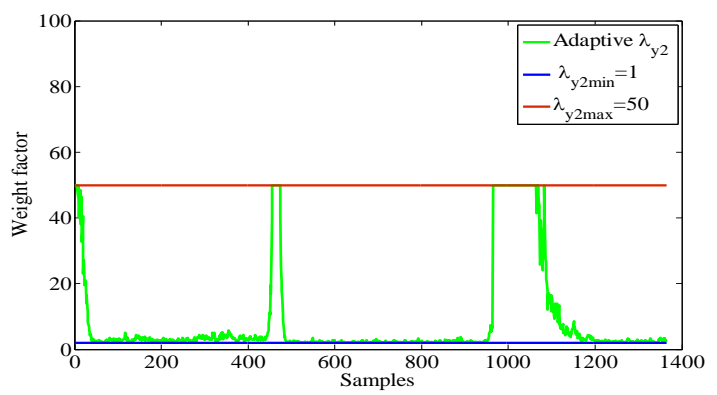

Fig 16: Evolution of the weighting factor $\lambda_{y 2}$.

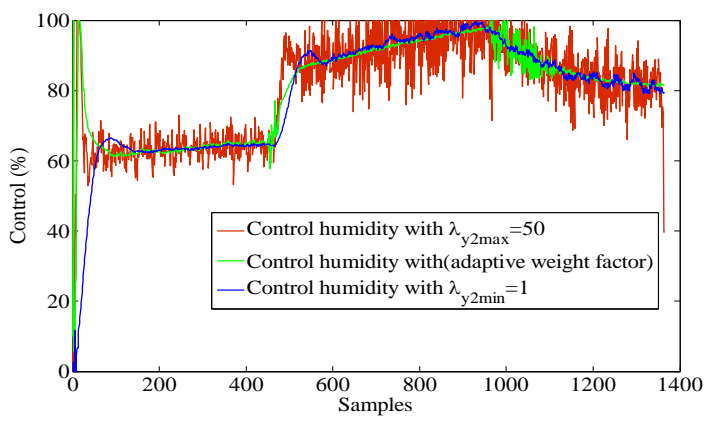

Fig 17: Simulation results of the temperature and humidity control in incubator system. 


\subsection{Experimental results and discussions}

The following experiment is conducted to investigate the performance of the proposed ADGPC (Adaptive Decoupling Generalized Predictive Controller) with adjusting of the weighting factors as in equation (35).

Fig 18 and Fig 19 show the step response of temperature and of humidity, respectively. One can see that the set-point change of temperature disturbs the humidity rate, which should be remained constant. This coupling effect can be minimizing by adjusting the error weighting factor, which increases at reference signal change of the other variable.

Fig 20 shows the real decoupled signal control of temperature and humidity with small, high and adaptive error weighting factor $\lambda_{y 2}$. Fig. 21 illustrates small $\lambda_{y 2 \min }=1$, high $\lambda_{y 2 \max }=20$ and adaptive error weighting factor $\lambda_{y 2}$. The adaptive error weighting factor is synchronized to the reference signal change. An increase of this factor generates a very drastic change in the manipulated variable (humidity control) which is an indicator of the decoupling effects. The advantage of using an adaptive error weighting factor is to keep a smooth control signal when the coupling effect is reduced.

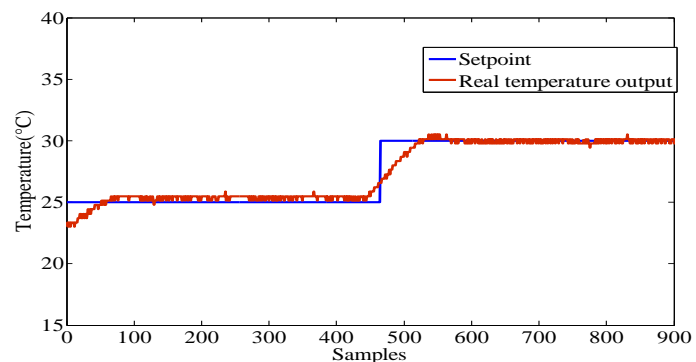

Fig 18: Actual close-loop step response of the temperature in incubator system.

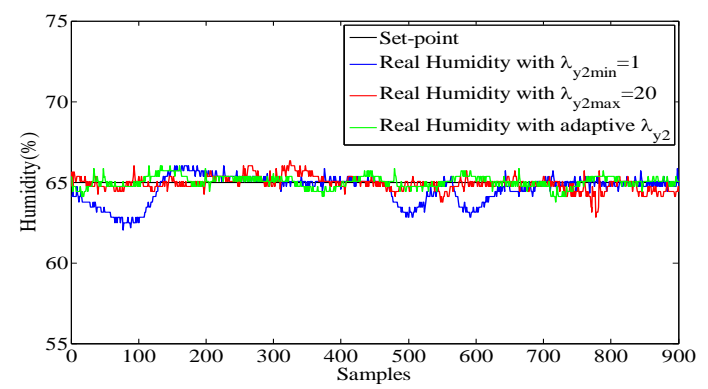

Fig 19: Actual close-loop step response of the humidity in incubator system.

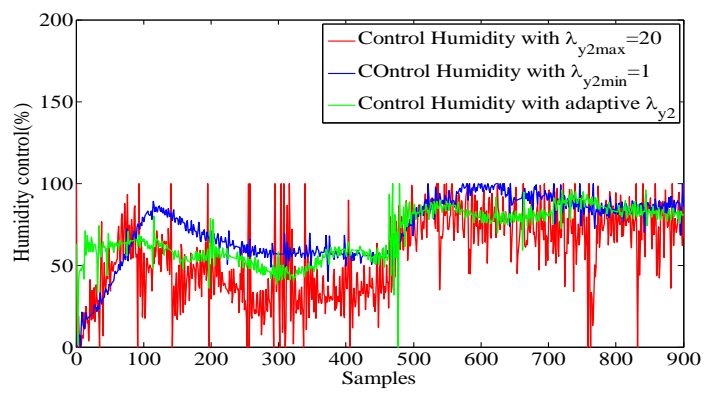

Fig 20: Experimental results of the real-time temperature and humidity control in incubator system.

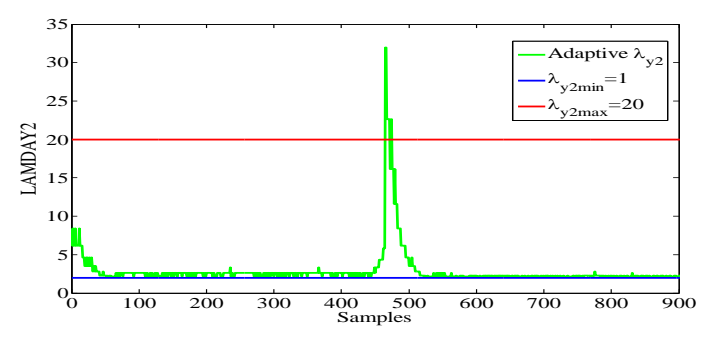

Fig 21: Evolution of the error weighting factor $\lambda_{y 2}$.

(a)

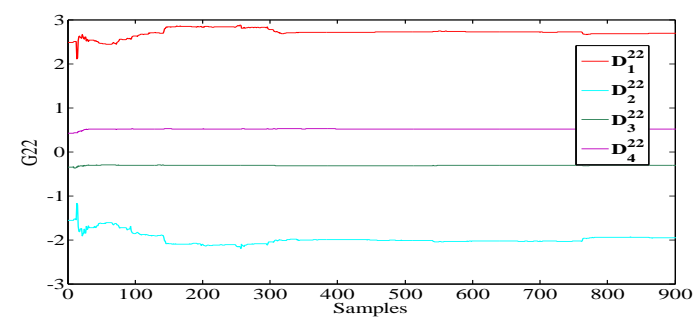

(b)

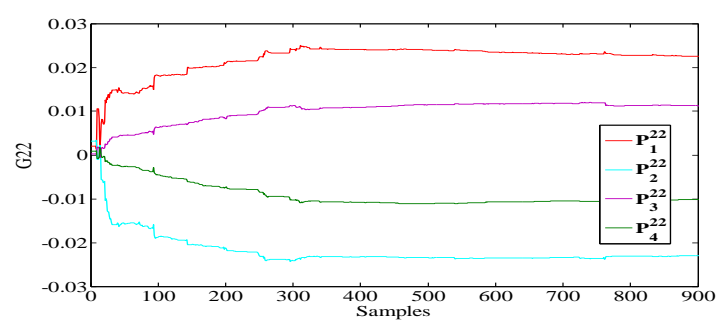

Fig 22: Convergent time histories of the eight estimated parameters in the real-time Humidity control experiment in incubator system.

(a)

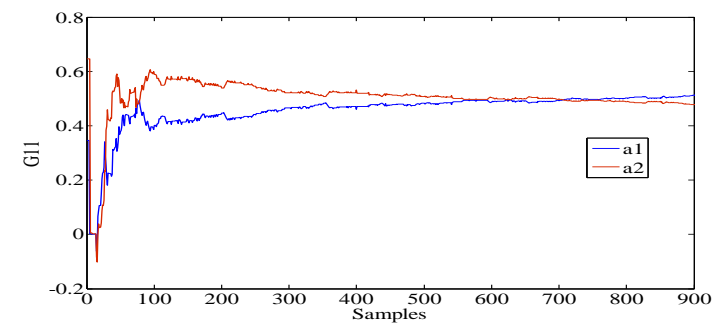

(b)

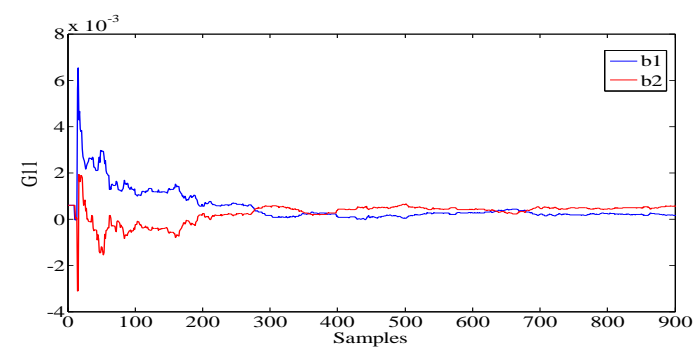

Fig 23: Convergent time histories of the four estimated parameters in the real-time Temperature control experiment in incubator system. 
Fig $22(a, b)$ and Fig $23(a, b)$, show the behavior of the estimated parameters in the real-time humidity and temperature control experiment in incubator system. Through the experimental results, the proposed adaptive decoupling generalized predictive control has been shown capable of giving satisfactory temperature and humidity tracking performance for the real incubator system. Also, we demonstrate that the decoupling by adaptive error weighting factor can almost eliminate the coupling influence with better control performance

\section{CONCLUSION}

This paper has developed a systematic design approach for an Adaptive Decoupling Generalized Predictive temperature and humidity Control (ADGPC) of an incubator process. Several decoupling methods have been demonstrated that the choice of a constant weighting factor on the output error does not allow at the same time to be powerful under control performance and decoupling. This compromise was resolved by varying the error weighting factor. In addition to estimate on line the unknown system parameters, we have used a MVRELS. The proposed ADGPC method is more general and practical than the approach presented by Schmitz et al. [15]. The simulation and experimental results have shown that the proposed control method is capable of giving satisfactory temperature and humidity control performance for the incubator system.

\section{REFERENCES}

[1] D. W. Clarke, C. Mohtadi and P. S. Tuffs, "Generalized Predictive Control - Part I and II”, Automatica, 23(2), pp. 137-160, 1987

[2] G. Li, D. P. Stoten and J.-Y. Tu, "Model predictive control of dynamically substructured systems with application to a servohydraulically actuated mechanical plant”, IET Control Theory Appl., 4(2), pp. 253-264, 2010.

[3] A. Ogunnaike and W. H. Ray, "Process Dynamics, Modeling, and Control”, Oxford Univ. Press, 1994.

[4] Bego, O. Peric, N. I. Petrovic, "Decoupling multivariable GPC with reference observation", 10th Mediterranean Electrotechnical Conference, vol.2, pp.819 - 822, 2000.

[5] P. R. Maurath, D. E. Seborg and D. A. Mellichamp, "Achieving decoupling with predictive controllers", Proc. Amer. Control Conf., Seattle, pp.1372-1377, 1986,

[6] F. Telliez, V. Bach, S. Delanaud, H. M. Baye, A. Leke, A. Apdoh and M. Abidiche, "Influence du niveau d'humidité de l'air sur le sommeil du nouveau-né en incubateur", RBM. Revue européenne de biotechnologie médicale, vol.21, pp.171-176, 1999.

[7] Gler and M. Burunkaya, "Humidity control of an incubator using the microcontroller-based active humidifier system employing an ultrasonic nebulizer",
Journal of Medical Engineering and Technology, vol.26, pp.82-88, 2002.

[8] D. W. Clarke, "Application of Generalized Predictive Control to Industrial Processes", IEEE Control System Magazine, vol. 8, pp.49-55, 1988.

[9] J. Richalet, D. O'Donavan, "Elementary Predictive Functional Control", Springer Verlag, Berlin, 201-105, 2009.

[10] J. T. J. Richalet, A. Rault and J. Papon, "Model predictive heuristic control: Applications to industial processes", Automatica, vol.14, pp. 413-428, 1978.

[11] C. C. Tsai and C. H. Huang, "Model reference adaptive predi- ctive control for a variable-frequency oil-cooling machine", IEEE Trans. Ind. Electron, 51,vol. 2, pp. 330$339,2004$.

[12] K. J. Astrom, "Theory and applications of adaptive control-A survey", Automatica, vol.19, pp.471-486,1983.

[13] D. M. Mathelin and R. Lozano, "Robust adaptive identification of slowly time-varying parameters with bounded disturbances", Automatica, vol.35, pp.12911305,1999

[14] M. A. Zermani, E. Feki and A. Mami, "Application of Adaptive Predictive Control to a Newborn Incubator", American J. of Engineering and Applied Sciences, 4 (2), pp. 235-243, 2011.

[15] U. Schmitz, R. Haber, F. Arousi and R. Bars, "Decoupling predictive control by error-dependent tuning of the weighting factor". AT\&P Journal PLUS 2 , pp.131-140, 2007.

[16] L. Zhang and R. Zhou, "Design of an incubator for premature infant based on labview", Conf Proc IEEE Eng Med Biol Soc, vol.4, pp.4079-4082, 2005.

[17] Drager Medical Systems, "Drager isolette c2000 infant incubator brochure", April 2004.

[18] J. L. Costa, C. S. Freire, B. A. Silva, M. P. Cursino, R. Oliveira, A. M. Pereira and F. L. Silva, "Humidity control system in newborn incubator", XIX IMEKO World Congress Fundamental and Applied Metrology, 2009.

[19] M. A. Zermani, E. Feki and A. Mami, "Application of Genetic Algorithms in identification and control of a new system humidification inside a newborn incubator", International Conference on Communications, Computing and Control Applications, pp. 1-6, 2011.

[20] A. K Abbas and S. Leonhardt, "System Identification of neonatal incubator based on adaptive ARMAX technique", IFMBE Proc, vol.22, pp.2515-2519, 2009.

[21] A. Hagenblad and L. Ljung, "Maximum likelihood identification of Wiener models with a linear regression initialization", Proc. 37th IEEE Conference on Decision and Control, USA, 1998 